\title{
Los grupos yumanos de Baja California: ¿indios de paz o indios de guerra? Una aproximación desde la teoría de la resistencia pasiva \\ Everardo Garduño*
}

\section{Resumen}

Para el prestigiado historiador Miguel LeónPortilla, los yumanos de Baja California no eran indios de guerra, sino indios de paz. En la opinión de este investigador, esto obedeció a que viviendo en un paleolítico fosilizado, estos grupos indígenas no presentaron mayor resistencia a la colonización europea y permitieron su dominación y posterior exterminio o asimilación. El presente trabajo cuestiona estas afirmaciones, no sólo por su imprecisión técnica, sino por el desconocimiento del papel de agencia que tuvieron los yumanos durante el periodo misional. Por el contrario, este artículo coincide con la afirmación de Edward Spicer (1962) en el sentido de que la resistencia tuvo lugar incluso entre aquellos grupos que no se vieron comprometidos en combates significativos en contra de los conquistadores. Se trata, en el caso particular de los yumanos, de una resistencia en contra del proyecto de Pueblo Indio y sus implicaciones en términos de sedentarización, trabajo agrícola y adopción de un esquema de autoridad central, que se contraponía a la naturaleza nómada de estos indígenas, como cazadores y recolectores organizados en un sistema segmentario de linajes. Las formas de resistencia yumana que aquí se describen,

\begin{abstract}
For the prestigious Mexican historian, Miguel León-Portilla, the Yumans of Baja California were not Indians of warfare, but rather Indians of peace. In the opinion of this researcher, it was because lived in the fossilized-paleolithic stage that these Indigenous groups did not present any kind of resistance against the European colonization, making possible their easy domination and posterior diminishment or assimilation. This paper questions these advancements not only because of their lack of technical precision, but also because they obscure the role of agency that these Indians played during the missionary period. On the contrary, this paper endorses Edward Spicer (1962) assertion that resistance was present even among those groups that were not seriously engaged in significant fights against the Spanish conquerors. In the particular case of the Yuman people, this is a resistance challenging the Pueblo Indio project and its related implications in terms of sedentary lifestyle, agricultural economy and the adoption of a scheme of central authority. As we know, these patterns were opposed to those observed among the Yumans as nomads, hunters and gatherers, organized into a segmentary lineage system. Moreover, the kind
\end{abstract}

* Doctor en Antropología Sociocultural. Investigador del Centro de Investigaciones Culturales-Museo, Universidad Autónoma de Baja California. Correo electrónico: everardo.garduno@uabc.edu.mx 
constituyen prácticas que forman parte de lo que James C. Scott (1990) denomina los "transcriptos ocultos y cotidianos" como la ingenuidad, la inteligencia encubierta de ignorancia disimulada y la ironía, así como la práctica económica y la práctica social estudiada por Jan Rus (1995), y que incluye la movilidad y la incorporación del sitio misional con propósito de permanencia y de reproducción.

Palabras clave: indígenas, Baja California, resistencia, colonización, yumanos. of resistance described in this paper constitute what James C. Scott (1990) refers as the hidden and daily life transcripts, such as ingenuity, intelligence simulating ignorance, and irony, as well as, those economic and social practices studied by Jan Rus (1995), which include mobility and appropriation of the missionary site, all with the intention of perpetuating the presence of these Indigenous people and make possible their social reproduction.

Keywords: indians, Baja California, resistance, colonization, yumans.

\section{Introducción}

Siguiendo la clasificación jesuítica de los indígenas del norte de México, León Portilla (1985) considera que los yumanos del desierto bajacaliforniano no eran indios de guerra, sino indios de paz. De acuerdo con este prestigiado investigador, esta caracterización corresponde a aquellos grupos que viviendo en un estadio de paleolítico fosilizado, no presentaron mayor resistencia a la colonización europea y permitieron su dominación y posterior exterminio o asimilación. Y es que a decir de este investigador, la ausencia de una beligerancia activa por parte de los yumanos, hizo posible que la conquista irrumpiera abruptamente su milenaria adaptación al medio ambiente, produjera en ellos una serie de traumas biológicos, psicológicos y culturales, y los condujera posteriormente hacia la muerte o absorción por la vía del mestizaje.

El presente trabajo cuestiona estas afirmaciones y plantea que la señalada pasividad de estos indígenas constituye en sí, una estrategia de resistencia frente al proyecto colonizador. En este sentido, este artículo retoma la afirmación de Edward Spicer (1962:16) acerca de la resistencia como un fenómeno generalizado, incluso entre aquellos grupos que no se vieron comprometidos en combates significativos en contra de los conquistadores. Es particularmente relevante para este punto de vista, la propuesta de James C. Scott (1990) en el sentido de que la resistencia de los grupos supeditados a la dominación puede expresarse a través de dos formas: 
una abierta y pública, como son las protestas, los boicots, la invasión de tierras y las rebeliones; y otra encubierta y cotidiana, a través del rumor, el chisme, los cuentos tradicionales, las bromas, las canciones, los rituales, los códigos intragrupales, los eufemismos, y una buena parte de la cultura tradicional. Es importante también para este artículo la propuesta de Jan Rus (1995) acerca de la práctica económica y la práctica social como formas de resistencia pasiva que se materializa a través de estrategias de aprovechamiento de recursos y de movilidad, e incluso a través de la incorporación de elementos introducidos o impuestos desde la misma hegemonía. Por último, este trabajo demuestra que la resistencia de los yumanos al sistema misional no se limitó a su forma encubierta y cotidiana descrita por Scott, sino que incluyó la rebelión y el boicot.

\section{¿Quiénes son los yumanos?}

Los yumanos son una familia etnolingüística a la cual pertenecen 15 grupos étnicos, distribuidos a lo largo de los desiertos de Baja California y Sonora en México, y Arizona y California en Estados Unidos (figura 1). La antigüedad de esta cultura en la parte norteamericana se ubica entre los 25 y 15000 años, mientras que en Baja California la presencia de estos grupos va solamente de los 2500 a los 150 años. Por esta razón, los especialistas han inferido que la presencia de estas poblaciones en la península es el resultado de una serie de migraciones que tuvieron lugar de norte a sur en periodos muy diferentes, dependiendo de la región de destino. Por ejemplo, Roger Owen (1959) afirma que los yumanos empezaron a arribar a la parte sur de la sierra de Juárez hace 2000 años, y continuaron haciéndolo hasta hace 600; Erlinda Burton (1973) sugiere que los habitantes del Valle Imperial, en Estados Unidos, han vivido en esa región desde hace 1500 años, y hace 400 empezaron a incursionar hacia el sur de dicho territorio; por último, Jesús Ángel Ochoa (1979) habla de inmigraciones indígenas históricas en las áreas contiguas a la Sierra de San Pedro Mártir, con una antigüedad de apenas 150 años.

Con esta antigüedad, los yumanos difícilmente pueden ser considerados los primeros pobladores de Baja California, aunque sí, el grupo prehistórico más relevante en varios aspectos -como veremos enseguida, los yumanos llegaron a observar un mayor desarrollo tecnológico y una 
Figura 1

Distribución geográfica de la familia etnolinguística yumana

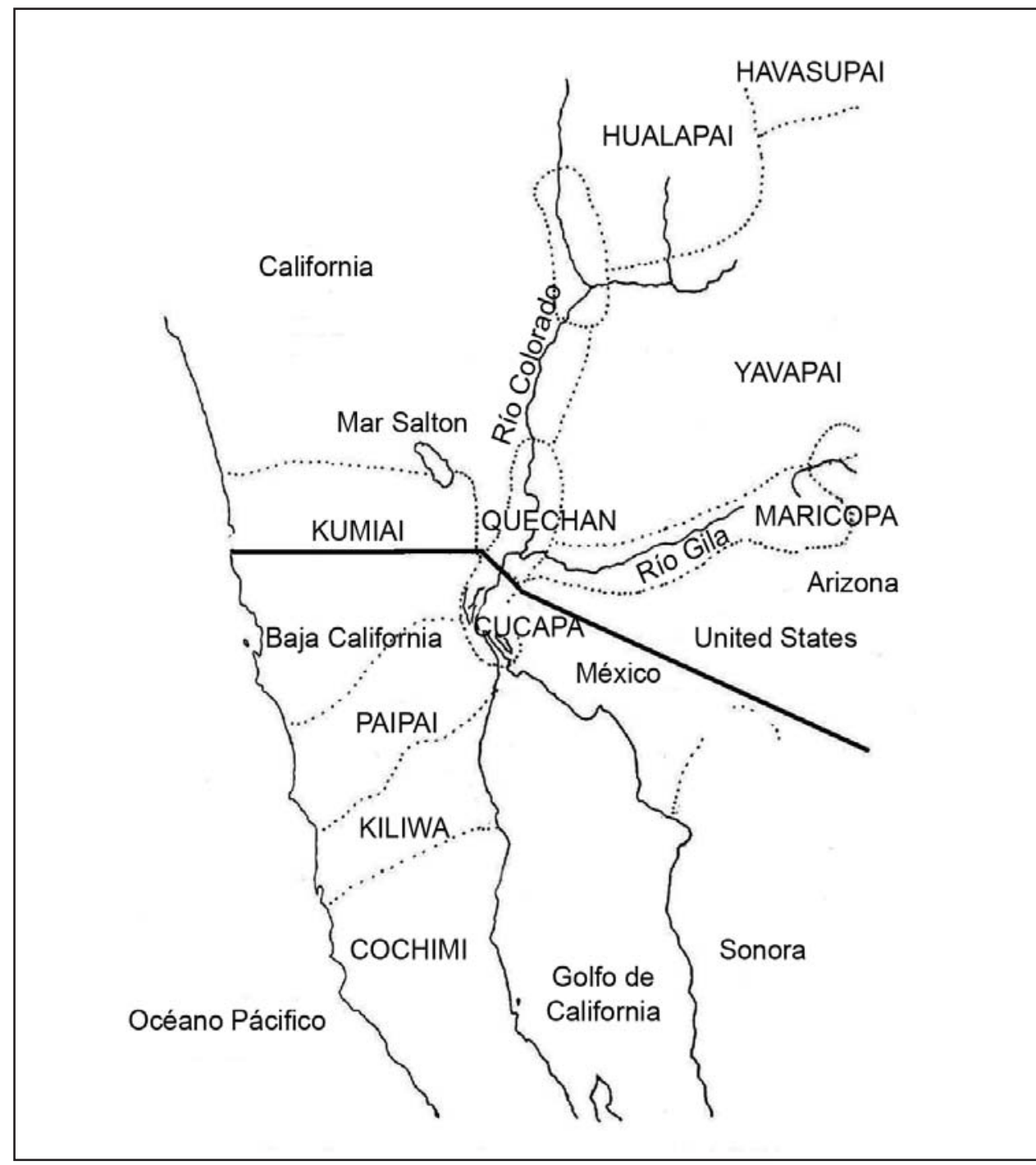

Fuente: Tomado de Ensenada, Nuevas Aportaciones para su Historia. Mexicali. UABC, 1999 y modificado para su publicación. 
mayor complejidad social, una presencia geográfica más amplia, una identidad territorial más definida, y una capacidad superior de adaptación, supervivencia y desarrollo local, que sus predecesores los grupos sandieguito y lajollano- ${ }^{1}$ Como es ampliamente sabido, esto hizo posible que los yumanos fueran el único grupo de la región que trascendió del prehistórico tardío hasta la actualidad.

Respecto a la cultura material, los sandieguito y los lajollano desarrollaron respectivamente una tecnología basada en la lítica y el empleo de la concha, mientras que los yumanos incorporaron la cerámica, la cestería y la cordelería. A decir de los arqueólogos, estas técnicas permitieron que estos últimos alcanzaran una mayor capacidad de almacenamiento de agua y semillas que los grupos preyumanos, y con ello, la posibilidad de establecer asentamientos más permanentes que sus predecesores, condición necesaria para desarrollar un cierto tipo de división del trabajo, una incipiente estratificación social y una presencia territorial más amplia: mientras que los antiguos sandieguito y lajollano limitaron su presencia a las sierras y a las costas, respectivamente, los yumanos poblaron tanto las costas como las sierras, los desiertos y los valles del norte peninsular.

Por otra parte, asociado a la amplia distribución geográfica de los yumanos a lo largo de los diversos ecosistemas del suroeste norteamericano y noroeste de México, se encuentra un proceso de distribución etnolinguística que dio como resultado el surgimiento de por lo menos 15 lenguas diferentes que representaban a un igual número de grupos étnicos con identidades estrechamente vinculadas a su territorialidad. Expresión de esto son los diferentes estilos de arte rupestre: como lo constatan Hedges (1975), y Bendímez y Laylander (1986), las representaciones realistas en el Desierto Central y los diseños abstractos del norte; las figuras antropomórficas y zoomórficas de las zonas montañosas y los petroglifos con diseños geométricos del desierto; los sitios arequeoastronómicos en el norte de la Sierra de Juárez y los geoglifos del desierto del Colorado en la parte norteamericana, representan todos en su conjunto marcas étnico-territoriales que funcionaban como delimitaciones fronterizas al interior de estos grupos indígenas.

\footnotetext{
1 La antigüedad de estos grupos es estimada en 10000 años para los sandieguito, y siete mil años en el caso de los lajollano.
} 
Más aún, pese a su mayor capacidad de asentamiento frente a los grupos preyumanos, los yumanos tampoco desarrollaron sociedades sedentarias, urbanas, agrícolas y regidas por una autoridad central o Estado, como en el centro y sur de México. Por el contrario, estos grupos desarrollaron un estilo de vida seminómada, con una organización fragmentaria caracterizada por la presencia de múltiples bandas y linajes en latente estado de conflicto, y un esquema de subsistencia orientado a la cacería, la pesca y la recolección. No obstante, lejos de constituir un signo de subdesarrollo o primitivismo que limitara las capacidades de supervivencia de estos grupos, este estilo de vida denotaba un profundo conocimiento del desierto y una sofisticada adaptación a las condiciones ambientales de la península, caracterizadas por la aridez y el clima extremoso. Tal y como lo explica Aschmann (1959), el carácter nómada de los yumanos, su organización fragmentaria y el conflicto, eran la condición y el mecanismo necesarios para activar la dinámica fisión-fusión de estas poblaciones, que se correspondía respectivamente con la necesidad de dispersión en tiempos de escasez, y la necesidad de potencializar esfuerzos para la caza y la recolección, en tiempos de abundancia. Pero además, signo inequívoco de adaptabilidad por parte de los yumanos a su circunstancia ambiental, constituye su capacidad de desarrollar una actividad agrícola importante, en las proximidades del Río Colorado. Frente a la generalizada idea de que la agricultura de los yumanos en estas latitudes era incipiente, fray Eusebio Francisco Kino (1701) nos dice que "todo este camino fue por una mera campiña de fertilísimas tierras, de hermosísimas milpas muy bien cultivadas con muchos maíces, frijolares y calabazales y con grandísimas tasajeras de tasajos de calabaza, que este género les dura después todo el año”.

Por último, otra muestra irrefutable de la gran capacidad de adaptabilidad de estos grupos a la circunstancia ambiental es el hecho de que mientras los sandieguitos y lajollanos habían ya desaparecido a la llegada de los españoles, los yumanos aún sobrevivían. Incluso podemos afirmar que más allá de su profundo conocimiento del desierto, estos indígenas demostraron una gran adaptabilidad a las condiciones sociohistóricas, al ser el único grupo que proveniente del prehistórico tardío, sobrevivió al impacto de la colonización europea y trascendió hasta la actualidad. Los grupos étnicos que pertenecen a esta familia etnolingüística y que hoy en día residen en alguna región del norte de Baja California son: los cucapá, 
Figura 2

Localización actual de las comunidades yumanas en Baja California

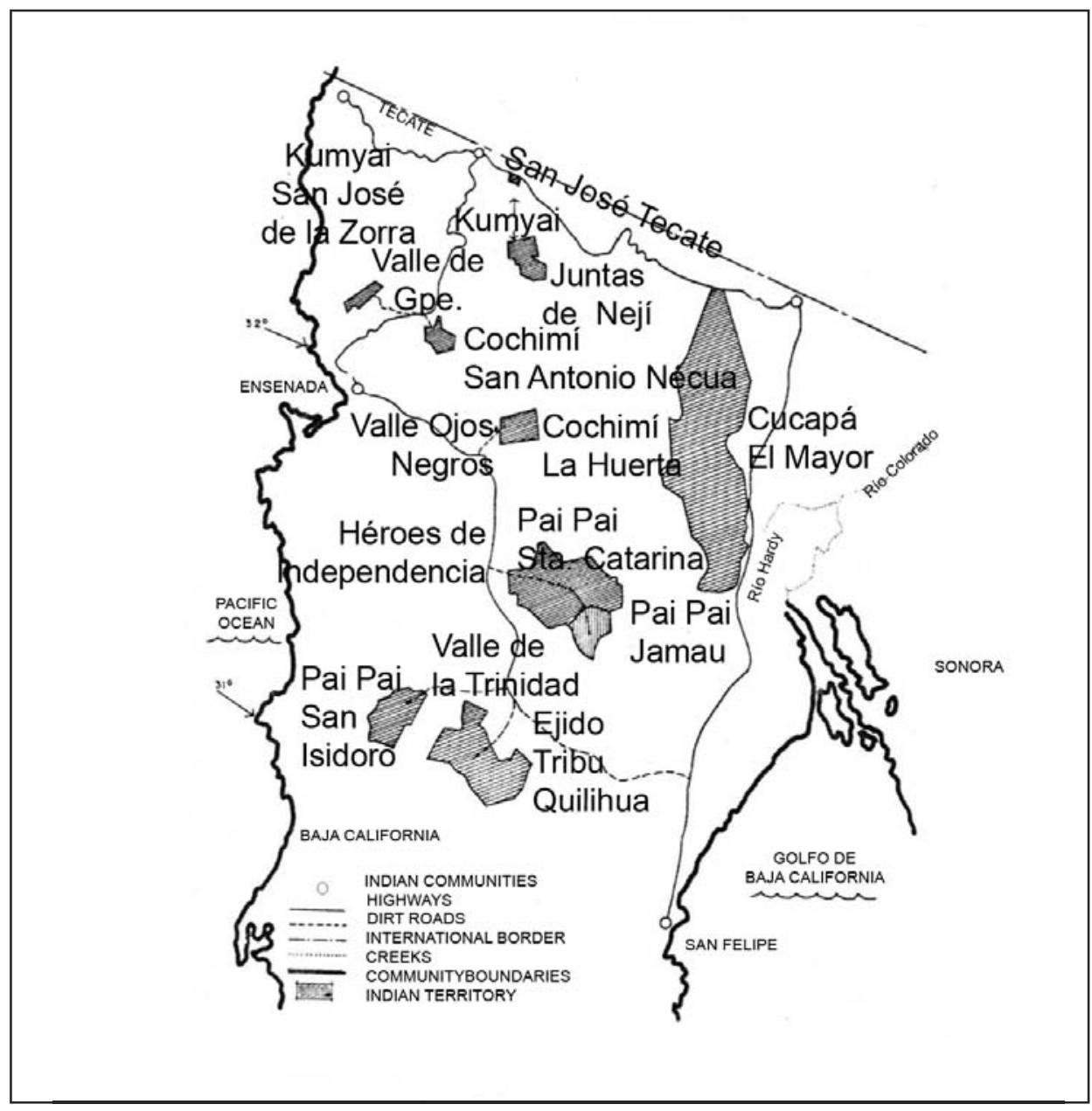

Fuente: Elaborado originalmente por la Escuela de Arquitectura de la Universidad Autónoma de Baja California, y modificado por el autor para su publicación. 
identificados también como "rieños" o "rianos", por habitar en las márgenes del Río Colorado; los kumiai y los tipai que viven en las mesetas costeras orientadas hacia el océano Pacífico; y los pai-pai y los kiliwa, conocidos también como "serreños", por residir respectivamente en los alrededores de la sierra de Juárez y de San Pedro Mártir (figura 2).

\section{La imposición del Pueblo Indio}

De acuerdo con Spicer (1962), los colonizadores europeos en el norte de México trataron de imponer sobre los grupos indígenas su religión, su lenguaje y, sobre todo, su noción de comunidad. Se trataba del proyecto de pueblo indio, el cual había sido introducido y desarrollado exitosamente entre los grupos agrícolas, sedentarios y urbanos del centro y sur de la Nueva España (Spicer, 1962:5). Siguiendo este modelo, los misioneros intentaron organizar a las bandas de cazadores y recolectores nómadas de Baja California, en una serie de poblaciones sedentarias establecidas alrededor de una cabecera, o pueblo principal, en donde estuviera localizada la misión como unidad productiva autosuficiente. ${ }^{2}$ La misión se establecía entonces en una zona en cuyos alrededores se hallaba un abundante número de indígenas, y en donde hubiera disponibilidad de agua y tierra fértil para desarrollar la actividad agrícola y pastoril.

No obstante, como sabemos, este modelo no resultó exitoso en la península debido a varios factores. Ciertamente, las particularidades del medio físico geográfico fueron uno de esos factores, así como la turbulencia

\footnotetext{
${ }^{2}$ Es importante mencionar que la colonización europea en la península de Baja California, tuvo lugar entre el siglo xvir y el siglo xix, con la presencia de los Jesuitas, Franciscanos y Dominicos. Sin embargo, debemos señalar también que la presencia de los primeros tuvo lugar entre 1697 y 1768, y se limitó únicamente al sur y centro peninsular, entre las poblaciones pericu, guaycura y cochimí; por su parte, los segundos permanecieron en Baja California solo por un corto tiempo (1767-1768), ocupando las misiones jesuitas del Desierto Central, entre los cochimí; finalmente, los Dominicos fueron los únicos que desarrollaron un contacto regular con los yumanos del norte, a lo largo de sesenta años, entre 1774 y 1834. Por esta razón, este artículo se refiere a algunos casos de jesuitas trabajando entre los yumanos peninsulares del Desierto Central, pero principalmente al período dominico.
} 
política y la crisis económica en vísperas de la independencia de México; más aún, factores decisivos en la cancelación del proyecto misional fueron las críticas por parte de las autoridades civiles y el descontento de los mismos aborígenes hacia los métodos empleados por los misioneros para desarrollarlo.

El primer paso en el desarrollo de este proyecto era reunir a las bandas indígenas en el sitio misional e iniciar su transformación en gentiles. Tal y como lo describen algunos historiadores, este proceso significó para estos grupos un cambio radical en su estilo de vida que incluyó la adopción forzada de la agricultura, la crianza de animales, la sedentarización en asentamientos compactos, de un sistema de gobierno central con estructura jerárquica, pero sobre todo, este cambio implicó la forzosa observancia de una rutina estricta, así como el padecimiento de castigos severos (Ochoa, 1976). Como se demuestra en la siguiente carta que la Junta de Fomento de los Territorios de la Alta y Baja California envió al gobierno nacional el 6 de abril de 1825, el sistema misional implicaba la ausencia notoria de un mínimo respeto a la integridad de los indígenas, ya que

[...] para llegar a ser cristianos, los gentiles deben renunciar a su entero derecho a su natural independencia. Desde el momento en el que se presentan para ser bautizados, deben mantenerse subordinados a las leyes monásticas [...] los neófitos deben continuar así, sin esperanza de obtener en su edad anciana los derechos civiles de la sociedad (Gómez, 1988:19).

Más aún, confirmando esta información se encuentra la acuciosa descripción que Manuel Clemente Rojo (1972:28-29) hiciera sobre la vida cotidiana de los indígenas al interior de una misión. Esto, sobre la base de los testimonios obtenidos directamente de Tomas Mancilla (el último misionero), los últimos soldados e indígenas sobrevivientes de las misiones, de María García y de Janitin (capitán kumiai), a ocho años de que la última misión cerrara sus puertas (1848):

Los indios en las misiones de la frontera viven sin libertad, despojados de todos los placeres de la vida, y obligados por la fuerza a trabajar sin ninguna recompensa. Una hora después del amanecer, los indígenas era obligados a levantarse ya fuera en el invierno o en el verano, para ser llevados a la iglesia 
en donde cantaban oraciones y decían algunos devotos rezos, palabras que aprendieron a repetir sin que la mayoría de ellos entendiera su significado.

Después de los ejercicios matutinos, los indios iban a la cocina de la iglesia a beber jarras de atole de maíz que había sido preparado para ellos, e inmediatamente después eran conducidos a los campos de labor de la misión, bajo la supervisión de una jefe o mayordomo que dirigía el trabajo y asignaba a cada uno las tareas que debían realizar [...] Quien no completara la tarea que le fuera encomendada por el mayordomo, sufría un castigo que consistía en una serie de 12 latigazos, mientras permanecía atado a un palo que había sido puesto en cada misión con este propósito y el cual era conocido como "La estaca”. Después del castigo, el indígena era llevado a la iglesia a continuar rezando. Cuando terminaba, era encerrado en una celda especial que correspondía a su estado civil. Los casados dormían separados de sus esposas en un lugar destinado para cada uno, los solteros varones en otro lugar diferente al de las mujeres solteras. El misionero conservaba las llaves de estas celdas.

Finalmente, sin lugar a dudas el periodo misional significó para los indígenas un sistema de sujeción por dependencia, explotación y exterminio, así como el despojo y degradación de su territorio. Esto alteró la movilidad tradicional de los yumanos y sus esquemas tradicionales de supervivencia, impactando drásticamente su reproducción biológica y social. No obstante, es una realidad que estos grupos indígenas reaccionaron y respondieron a estas acciones por medio de una serie de actos que fueron regularmente calificados por los misioneros, las agencias de gobierno e incluso los investigadores como indolencia, vandalismo o desorganización generalizada. En mi opinión, se trataban todos ellos de acciones de resistencia pasiva, e incluso, en algunas ocasiones, de resistencia activa.

\section{Las formas de resistencia pasiva entre los yumanos}

Es ampliamente sabido que uno de los principales obstáculos con los que se encontraron los misioneros en Baja California para desarrollar su proyecto de Pueblo Indio fue la actitud de los indígenas frente a la disciplina y el trabajo en la misión, calificada por los colonizadores como indolencia y negligencia. En el multicitado texto de Baeggert (1942), por 
ejemplo, encontramos una serie de epítetos que sin lugar a dudas describen la mirada descalificadora y llena de frustración, con la que los misioneros veían a los indígenas. No obstante, una segunda lectura a este mismo texto nos puede arrojar elementos para sugerir que dicha actitud podía constituir parte de lo que Scott (1990) denomina "resistencia oculta y cotidiana a la dominación". Como recordaremos, en 1772, en referencia a los yumanos peninsulares, Baeggert afirma que debido a la desobediencia, el hurto y la intriga, era difícil para los misioneros establecer absoluto control sobre estos indios. A decir de este misionero, estos indígenas son "insolentes, ingratos, mentirosos, vulgares, perezosos en extremo y grandes charlatanes, en fin, gente que no puede ser dominada" (1942:109).

Por otra parte, existen también indicios en el sentido de que los yumanos llegaron a integrar a la ingenuidad, la inteligencia encubierta de ignorancia disimulada y la ironía como parte de su repertorio de resistencia pasiva, particularmente frente al esquema de autoridad central implícito en el proyecto del colonizador. Esto es ilustrado por el siguiente testimonio del padre Garcés de San Bernardino de los Opas, quien describe un evento ocurrido en la jornada del 14 de noviembre de 1772: "Sugerí también a los ancianos reunirse para llevar a cabo el nombramiento de un capitán entre ellos, y en el nombre del rey, un gobernador y mayores; en respuesta, un anciano me dijo seriamente estas palabras: 'mira, la justicia es para castigar lo malo, y no siendo malos nosotros, ¿para qué es la justicia?” (Ochoa, 1976:18). De igual forma, Mauricio Mixco (1983:197) presenta el caso de un anciano kiliwa, quien después de haber sido nombrado con el título de general, tomó el nombramiento con sus manos y preguntó: "En donde voy a meter esto? Estaba desnudo, no tenía bolsas".

Otro tipo de resistencia pasiva entre los yumanos es aquella estudiada por Jan Rus (1995) entre los indios tzeltales de Chiapas, la cual consiste en el desarrollo de una movilidad con patrones de frecuencia, distancia y circularidad siempre acorde a la intensidad de la crisis económica que la impulsa. Como veremos enseguida, pese a su resistencia al sistema misional, los yumanos adoptaron en los momentos de mayor escasez una movilidad circular anual que incluía a estos asentamientos como estaciones temporales. De acuerdo con Magaña (2003), aun en estado de libertad, estos indígenas continuaron regresando a la misión anualmente con propósitos de subsistencia y de reproducción del grupo. De acuerdo con este 
autor, durante los periodos de mayor abundancia de alimento silvestre, la primavera, el verano y el otoño, los yumanos deambulaban entre los desiertos, las costas y las sierras. Sin embargo, durante el periodo de mayor escasez, el invierno, estos indígenas acudían a la misión. Evidencia de este hecho puede ser encontrado en los cuadernos de 1775-1800 de la misión de Santo Domingo, en los cuales los bautizos, matrimonios y decesos fueron registrados en mayor frecuencia después de octubre, mientras que el registro de estos eventos disminuía de marzo a julio y de septiembre a octubre; esto es, justo cuando estaba teniendo lugar la colecta de moluscos y la colecta del piñón y la bellota, respectivamente.

Pero además, para Magaña la frecuencia observada en los registros misionales no estaba solamente asociada a la ausencia y presencia de los yumanos en el sitio misional, sino también a su actividad reproductiva. Desde el punto de vista de este autor, el incremento de la frecuencia de bautizos registrado en la misión durante el invierno, supone la existencia de una actividad sexual previa. Dado que los hombres y las mujeres indígenas permanecían separados forzosamente durante su estancia en la misión, es fácil suponer que dicha actividad tenía lugar entre ellos durante el periodo en el cual se encontraban fuera de este sitio, esto es, entre la primavera y el verano, y durante el otoño, y que una vez en espera de la descendencia, acudían al sitio misional en búsqueda de alimento y abrigo que asegurara la sobrevivencia de los nuevos miembros de la tribu.

Sin lugar a dudas, estos eventos revelan la capacidad de adaptación de los yumanos a las nuevas circunstancias y de incorporación de elementos introducidos e incluso impuestos por los colonizadores, en su propio beneficio; también, estos hechos ilustran sobre una particular sensibilidad de estos grupos para mantener una continua negociación con el colonizador y desarrollar una vida seminómada que abarcara tanto su territorio tradicional como el sitio misional; todo esto, con propósitos reproductivos, es decir, con propósitos de permanencia. Es por ello que estos eventos deben ser interpretados como parte del esquema de la resistencia pasiva caracterizada por Falcón (2002:84) como aquellas “acciones pequeñas, ocultas y cotidianas, a través de las cuales los pobres y marginados, negocian individual o colectivamente su lugar dentro de la estructura de poder, evadiendo cualquier confrontación directa con los poderosos económicamente y la autoridad". 


\section{La resistencia activa y sus propósitos}

Por otra parte, si bien la resistencia de estos indígenas fue en general pasiva, la crisis estructural en la Nueva España hizo que ésta se transformara en un tipo de resistencia activa. Como es sabido, esta crisis estructural estuvo marcada por la turbulencia política y la crisis económica, resultado de las guerras de independencia, lo cual hizo imposible que los misioneros retuvieran dentro del sitio misional tanto a los indígenas como a los soldados que guardaban de su seguridad. En ese contexto, tal y como lo expresa el siguiente testimonio, los indígenas bautizados escaparon a las montañas retornando a su vida nómada, desde donde empezaron a organizar acciones de merma mediante el hurto en contra de las misiones:

Los niños de tales madres pasan los años enteros sin probar tortilla y beber sotol, y comiendo solo ratas y agaves sobreviven como pueden. Sus padres misioneros han confrontado grandes conflictos para obtener recursos para alimentarlos, pero inevitablemente ellos escapan y se dispersan, y hambrientos van por las montanas y las playas, y lejos de avanzar en religión y civilización, van hacia atrás cada día, hacia una atroz conducta, conservando e incluso progresando en la ciencia del latrocinio (González, 1825:3).

Más aún, una vez libres y fuera de todo control de los misioneros, estos prófugos y evangelizados indígenas establecieron contacto con los indígenas no bautizados de diverso origen, y a pesar de su estatuto de gentiles que indicaba su transformación a la civilización occidental, asumieron entre estos últimos el liderazgo para conducirlos a atacar a las misiones. Evidencia sobre este tipo de acciones se encuentra en el manuscrito de 1824, perteneciente al señor Arguello:

Habiendo tenido algunas particulares noticias sobre como los indios cristianizados de las misiones dominicas de la antigua frontera de California, junto con algunos otros indios de la misión de San Diego y gente de aquellas sierras y de las márgenes del Río Colorado, estaban produciendo serios daños al ganado de las misiones y a los ranchos privados, robando los caballos domesticados que comen y venden a aquellas tribus que habitan la otra margen del río, los Indios cristianizados, salvajes y diabólicos cuya presencia ha llegado a 
ser muy fuerte y quizás incrementada con la compañía de la gentilidad que vive en las sierras y habita las márgenes del Río Colorado, y con otras tribus de tierras desconocidas para nosotros (Arguello, 1824).

Incluso, como podemos ver en un tercer manuscrito, la presencia amenazadora de los indígenas frente a las misiones no era tan insignificante como lo asume Miguel León-Portilla (1983). Por el contrario, tal y como lo afirma el Marqués de Vivanco en su carta al Ministro de Guerra y Marina, éstos sobrepasaban considerablemente la capacidad de defensa de los españoles en Baja California: "La gentilidad es mucha; las tropas son pocas, además del hecho de que están descontentas debido a la falta de sus salarios. Le doy cuenta de todo esto a mi Excelente Señor de Guerra y Marina, con el propósito de obtener recursos, ropa y avituallamiento de guerra, dado que todo se necesita en esta remota provincia" (Vivanco, 1824).

Finalmente, el conocido testimonio de una mujer indígena kumiai recogido por Rojo (1972:43) cuestiona a quienes consideran que estos ataques indígenas eran simplemente acciones vandálicas, resultado de la desorganización generalizada de estos grupos, y confirma la idea de la rebelión con propósitos claros. Estando cautiva en la misión de Guadalupe, María experimenta la abrupta irrupción de la quietud de este recinto, como resultado del ataque de un grupo numeroso de kumiais, cuyo líder le expresa lo siguiente: "No tengas miedo, no he ordenado matar a nadie, aunque mi gente haya matado. Al único que estoy buscando es al sacerdote, porque está bautizando por la fuerza a la gente de mi tribu, para esclavizarlos en la misión, justo en la forma en que tú estás ahora, sin disfrutar de tu libertad y viviendo como caballos".

\section{Los resultados de la resistencia indígena}

Sin duda, como ya se comentó anteriormente, pese a la existencia de estas estrategias de resistencia indígena, el impacto de la colonización en la vida de estos grupos fue dramático. Sin embargo, esto no significa que los actos de resistencia pasiva y activa, anteriormente descritos, no fueran exitosos en varios sentidos. Gracias a ellos, los misioneros cambiaron su plan original, lo cual hizo posible que los indígenas se reincorporaran periódicamente a su vida nómada y mantuvieran su sentido de colectividad. 
Mario Magaña (1999) documenta cómo después de la destrucción de las misiones de San Pedro, San Pablo y de la Purísima Concepción en 1781, la corona española empezó a declinar sus intenciones de colonizar Baja California. Esta decisión impidió, primero, que los misioneros establecieran una regular comunicación entre las misiones de Sonora y la Alta California; segundo, que establecieran control sobre el $50 \%$ del territorio; y tercero, que retuvieran de manera permanente bajo campana, a los indios evangelizados. Debido a estos eventos, los indios empezaron a ser conducidos a la misión únicamente para obtener instrucción religiosa; después se les permitía retornar a su antiguo estilo de vida y obtener sus alimentos por sí solos. Esta circunstancia hizo posible que las poblaciones nativas mantuvieran su acostumbrada movilidad a lo largo de su territorio tradicional, mantuvieran sus relaciones con aquellos grupos que habitaban territorios fuera del control de los misioneros, y reforzaran sus afinidades: i.e. los kiliwa y los pai-pai del Golfo de California, con los cucapá del Río Colorado.

Más aún, gracias a estas habilidades demostradas por los yumanos, una tercera o cuarta parte de su población sobrevivió a la colonización europea y retornó a su vida en las montañas, una vez clausurada la última misión. Esto es demostrado a través de correlacionar dos registros de campo realizados inmediatamente después de la partida de los misioneros. Se trata del censo realizado en 1880 por el general Luis E. Torres, jefe político y militar del Distrito Norte de Baja California, y el recorrido llevado a cabo en 1840 por David Golbaum. En el primero se establece que a la partida de los misioneros existían 4424 indígenas viviendo entre las sierras de Juárez y San Pedro Mártir, y las inmediaciones del Río Colorado (Martínez, 1985). En el segundo se constata que de este número, sólo unos cuantos permanecieron en los asentamientos exmisionales, ya que la mayoría se reagrupó en pequeñas bandas y regresó a su estilo de vida nómada. De acuerdo con Golbaum, de las misiones sólo quedaron vestigios: canales, cisternas, pequeñas represas para irrigar las tierras, unas cuantas casas de adobe con techos de ramas conformando una serie de pequeños e inestables caseríos, e incluso cuevas, en donde sólo alrededor de cien indígenas continuaban viviendo y practicando algunas actividades aprendidas a los misioneros, y algunas actividades correspondientes a sus propias estrategias de supervivencia: "Algunos practicaban agricultura a 
pequeña escala, la crianza de un poco de ganado y el curtido de cuero, mientras que la mayoría de ellos cazaba y colectaba plantas, buscaba miel silvestre, pescaba y elaboraba artefactos de cordelería, cestería y cerámica" (Golbaum, 1984:20).

Por último, acontecimientos documentados y testimonios obtenidos durante el siglo $\mathrm{xx}$, nos permiten afirmar que la resistencia presentada por estos grupos en contra del esquema de autoridad central permitió, aunque de manera oculta, la persistencia de su sistema fragmentario de organización social basado en linajes, y la presencia limitada, aunque pública, de los nuevos cargos.

Tal y como se comentó anteriormente, el modelo de Pueblo Indio y su esquema de autoridad central y jerarquizada, se confrontaba con el sistema segmentario de linajes de los yumanos. Por esta razón, los indígenas no cesaron de poner en tela de juicio los nombramientos, a los cuales si bien les dieron un lugar altamente visible desde fuera del grupo, nunca les otorgaron un poder real hacia el interior. Al respecto, Meigs (1939: 45-46) nos explica cómo ya avanzado el siglo xx, mucho tiempo después de que los misioneros habían sido expulsados de Baja California, estos grupos llegaron a ser identificados por las agencias del Estado a través de las figuras de capitanes y generales introducidos por los españoles; no obstante, éstas funcionaban sólo como figuras de representatividad frente al gobierno, ya que la autoridad legítima continuaba estando depositada en la cabeza del linaje. Dentro de la lógica de este esquema de autoridad, los capitanes y generales eran vistos como cabezas de linajes específicos y no como autoridades de todos los linajes, y menos, de los linajes enemigos. La persistencia e importancia del sistema segmentario de linajes que aparentemente había desaparecido durante el periodo misional, quedó al descubierto en una serie de eventos ocurridos en la primera cuarta parte del siglo xx. Como es ampliamente sabido, durante la Revolución mexicana, los hermanos Flores-Magón invadieron Baja California y la declararon la primera Republica Socialista del mundo. Como lo ha documentado Owen (1963), esto produjo una serie de confrontaciones armadas, en las cuales los indígenas participaron de una u otra parte. Algunos historiadores vieron en estos hechos un alineamiento ideológico de los grupos indígenas con alguna de las facciones en conflicto; otros simplemente denunciaron la introducción de conflictos ajenos al interior de los yumanos; para Owen 
(1963), sin embargo, se trató de la emergencia de la visibilización del viejo carácter semihostil del sistema de linajes. A decir de este autor, aquellos que se aliaron con las tropas federales para combatir a los magonistas pertenecían a los grupos que habitaban en el norte, particularmente a los kumiai y tipai de los linajes Koal, Miakwas y Qwashaqsh. Por su parte, quienes acompañaron a los magonistas en su marcha de Mexicali hacia Ensenada fueron los yumanos que habitaban en los alrededores del Valle de la Trinidad, los pai-pai de los linajes Kweljwat, Jwa(ch) y Jamsulch, y prácticamente todos los kiliwas, con excepción del linaje Jepi Pakawas, el cual se encontraba viviendo en ese entonces, entre los indios tipai. Como lo destaca Owen (1963), estas confrontaciones armadas no respondían a una identificación ideológica con una u otra facción del conflicto revolucionario, ni de la introducción de conflictos ajenos, sino más bien, representaban la oportunidad para tener acceso a armas de fuego y municiones con el propósito de dar salida a sus propias rivalidades interlinajes.

Owen (1963) argumenta que antes de que arribaran los magonistas, estos dos grupos de linajes habían entrado ya en tensión, como resultado de una serie de muertes ocurridas y adjudicadas a la acción maléfica de un chamán diabólico. Este chamán era Jorge González, quien pertenecía al linaje Qwashaqsh, y quien fuera muerto en medio de estas tribulaciones en las manos de los magonistas apoyados por el linaje Kulwat -parientes de las supuestas victimas del maléfico chamán (Owen, 1959:7; Álvarez, 1975: 67; y Mixco, 1983:9). De esta manera, el sistema segmentario de linajes encontró objetivación en estos hechos armados.

\section{Conclusiones}

¿Indios de paz o indios de guerra? Para Miguel León-Portilla la respuesta es fácil: los yumanos son indios de paz por haberse incorporado pasivamente a la colonización europea, y no haber ofrecido una resistencia activa frente a ésta. Para este artículo, sin embargo, la respuesta es más compleja, sobre todo si se considera que es posible resistir pasivamente e incorporarse a los procesos hegemónicos resistiendo. En este artículo hemos demostrado que los grupos yumanos constituyen un ejemplo de esta resistencia pasiva y contrahegemónica, y en no pocas ocasiones de resistencia activa, frente a la imposición de la noción de comunidad agrícola, sedentaria y 
territorialmente delimitada. Se trata en esencia de una resistencia que incluye: 1) acciones encubiertas como la ironía, la ignorancia disimulada, los eufemismos, el hurto y una actitud calificada por los misioneros como indolencia en el trabajo; 2) estrategias de supervivencia como la reelaboración de la movilidad tradicional; y 3) actos de rebelión y boicot. De esta manera, ante la pregunta inicial, podríamos afirmar que los yumanos fueron principalmente indios de paz, y excepcionalmente indios de guerra, pero no indios que aceptaron pasivamente el proyecto colonizador.

¿Indios de un paleolítico fosilizado que no sobrevivieron al contacto? Para Miguel León-Portilla (1985) la ecuación es clara: el estado primitivo de los yumanos constituyó una condición de elevada vulnerabilidad frente a los colonizadores europeos, que explica su falta de animosidad frente a éstos, sus traumas biológicos, psicológicos y culturales, y su paulatina desaparición. Para este artículo, sin embargo, esta ecuación es técnicamente desafortunada. Como bien lo han apuntado los especialistas, a diferencia de los antiguos grupos sandieguito y lajollano, los yumanos se caracterizaron por el desarrollo de la cerámica y en algunas regiones de la agricultura. Desde el punto de vista de la arqueología, estos dos componentes son precisamente los que constituyen la frontera del neolítico frente al paleolítico.

Más aún, los yumanos no sucumbieron frente a las duras condiciones de la naturaleza ni a la colonización europea. Por el contrario, estos grupos son un ejemplo de éxito adaptativo y de resistencia frente a ambas. A decir de los mismos especialistas, sus características de cazadores, recolectores y nómadas, combinadas con el desarrollo tecnológico basado en la cerámica, hicieron posible su poblamiento y supervivencia en prácticamente todo el norte de la península, incluyendo las regiones de mayor aridez como el Desierto Central. Asimismo, desde el conocimiento adquirido como grupos pertenecientes al prehistórico tardío, los yumanos son un ejemplo de resistencia contrahegemónica: se rebelaron frente a los nuevos constructos que desde la hegemonía les fueron impuestos, al tiempo que los incorporaron para ponerlos a disposición de sus estrategias de supervivencia y reproducción. Como aquí se ha demostrado, estos grupos se opusieron sistemáticamente al proyecto de Pueblo Indio, pero adoptaron al sitio misional como resguardo temporal para asegurar la preservación de su descendencia. Incluso, una vez cerrada la última de las misiones dominicas en el norte de Baja California, los yumanos reestablecieron su estilo de vida 
tradicional, reproduciendo únicamente aquella parte del legado misional que les resultó útil para su supervivencia. Esto explica, sin lugar a dudas, el porqué la presencia de los yumanos, teniendo su origen en la prehistoria, trascendió a la época moderna.

Con lo anterior, este artículo no pretende negar los cambios ocurridos en lo social, demográfico y cultural a los yumanos. Nadie puede negar que en la actualidad estos grupos residen en comunidades sedentarias que sobreviven principalmente de su trabajo como asalariados en los ranchos cercanos, que se encuentran subsumidos en la llamada "cultura del vaquero", y que en algunos casos es evidente la pérdida de sus rasgos étnicos distintivos como la lengua. Sin embargo, la pregunta final sería: ¿'Todas estas transformaciones son el resultado de la colonización europea, como lo suponen las apreciaciones de Miguel León-Portilla y el punto de vista generalizado? En este artículo hemos demostrado que existen evidencias suficientes para suponer que esto no es así. El propio censo de Miguel Torres y la exploración de Golbaum nos hablan de una significativa presencia de los yumanos al momento en que los dominicos dejaron Baja California. A esto podemos añadir los recientes hallazgos arqueológicos en la zona de vallecitos, en La Rumorosa, a través de los cuales es posible constatar la existencia de poblaciones nómadas sobreviviendo de la cacería, la recolección y la pesca, practicando las cremaciones tradicionales y la elaboración de atuendos de piel, en un periodo posterior a la llegada de los españoles. Esto sugiere, sin lugar a dudas, que las causas principales de las transformaciones antes señaladas deben buscarse hacia el siglo xx. No obstante, esto es objeto de otro artículo.

\section{Bibliografías}

Álvarez, Anita (1975), Los primeros pobladores de Baja California, Gobierno del Estado de Baja California, Mexicali.

Arguello, Luis Antonio (1824), Archivo General de la Nación Fondo, Gobernación Ref.: vol. 67 Sección S/S Exp.2 Cih 1824.14, periodo 1824, Centro de Investigaciones Históricas UNAM-UABC.

Aschmann, Homer (1959), The Central Desert of Baja California, Demography and Ecology, University of California Press, Berkeley.

Baeggert, Juan Jacobo (1942), Noticias de la Península Americana de California, Porrúa, México. 
Bendimez, Julie y Don Laylander (1986), El arte rupestre del norte de Baja California, Universidad Autónoma de Baja California, Mexicali.

Burton, Erlinda (1973), "Culturas prehistóricas en el Valle Imperial”, Calafia, vol. II, núm. 2, México.

Falcón, Romana (2002), México descalzo. Estrategias de sobrevivencia frente a la modernización, Plaza y Janés, México.

Golbaum, David (1984), "Noticia respecto a las comunidades indígenas que pueblan el Distrito Norte de la Baja California”, Calafia, núm. 3, México, pp. $3-5$.

Gómez, José Alfredo (1988), La época misional en Baja California, Museo Regional Universitario, Mexicali.

González, Pedro (1825), Archivo General de la Nación Fondo, Gobernación Ref: vol. 78 Sección S/S Exp.19 Cih 1825.1 C5 16, periodo 1825, Centro de Investigaciones Históricas UNAM-UABC.

Hedges, Ken (1975), "Pintura rupestre kumiai en las montañas septentrionales de la península de Baja California”, Calafia, vol. II, núm. 6, México, pp. 26-35.

Kino, Eusebio Francisco (1985), Crónica de la Pimería Alta. Favores celestiales. Gobierno del Estado de Sonora, Hermosillo.

León-Portilla, Miguel (1983), Los primeros californios: pre-historia y etnohistoria. Panorama histórico de Baja California, Universidad Nacional Autónoma de México/Universidad Autónoma de Baja California, Mexicali. (1985), "Los indígenas de la frontera entre México y los Estados Unidos sin reglas del juego y juego sin reglas en la vida fronteriza", en Mario Miranda y James Wilkie (eds.), Reunión de universidades fronterizas de México y Estados Unidos, Anuies, Profmex, México, pp. 35-50.

Magaña, Mario (1999), Indígenas, misiones y ranchos en el siglo xix en Ensenada”, en M. Samaniego y J. Martínez (eds.), Nuevas aportaciones para su historia, Universidad Autónoma de Baja California, Mexicali, pp. 81-113.

(2003), "Antes éramos libres. Antes era muy libre e íbamos ahí... Ensayo histórico sobre la movilidad en los indígenas yumanos de Baja California", Culturales, vol. I, núm. 3, México.

Martínez, Jorge (1985), Música indígena en Baja California, Instituto de Investigaciones Históricas, Universidad Autónoma de Baja California.

Meigs, Peveril (1939), The Kiliwa Indians of Lower California, University of California Press, Berkeley.

Mixco, Mauricio (1983), Kiliwa Texts. When I Have Donned My Crest of Stars in Anthropological Papers, University of Utah Press, Salt Lake City.

Ochoa, Jesús (1976), "Caciques, señores, capitanes y gobernadores. Nombramientos indígenas en Baja California”, Calafia, vol. III, núm. 3, México, pp.15-25.

(1979), "Distribución actual de los grupos indígenas de Baja California", Calafia, vol. IV, núm. 1, México, pp.10-18. 
Owen, Roger (1959), "The Indians of Santa Catarina, Baja California Norte, Mexico", Concepts of Disease and Curing Ph.D. dissertation, Los Ángeles, Department of Anthropology, University of California. (1963), "Indians and Revolution: The 1911 Invasion of Baja California, Mexico", Ethnohistory, vol. 10, núm. 4, Estados Unidos, pp. 373-395.

Rojo, Manuel Clemente (1972), [1879] Historical Notes on Lower California, Dawson's Book Shop, Baja California Travelers Series 26, Los Ángeles.

Rus, Jan (1995), “The Reordering of Native Society in Highland Chiapas, México, 1974-1994", European Review of Latin American and Caribbean Studies, núm. 58, Holanda, pp. 71-89.

Scott, James (1990), Domination and the Arts of Resistance. Hidden Transcripts, Yale University Press, New Haven y Londres.

Spicer, Edward (1962), Cycles of Conquest: The Impact of Spain, México and the United States on the Indians of the Southwest, 1533-1960, University of Arizona Press, Tucson,

Vivanco, El Marqués de (1824), Archivo General de la Nación Fondo: Gobernación Ref: vol. 67 Sección S/S Exp.2 Cih 1824.14, periodo 1824. Centro de Investigaciones Históricas UNAM-UABC.

Artículo recibido en marzo de 2010 Artículo aprobado en agosto de 2010 
